\title{
杂化钙钛矿光伏材料的合成及热稳定性研究
}

一推荐一个综合化学实验

车平 ${ }^{*}$, 杨涵, 范慧俐, 王明文, 李文军

北京科技大学化学系, 北京 100086

摘要: 介绍了一个综合性化学实验, 内容包括一步溶液法制备有机-无机杂化䥻钛矿光伏材料、材料结构与形貌表征、 吸光系数测定及热稳定性研究。

关键词：杂化钲钛矿；光伏材料；合成；热稳定性；综合实验

中图分类号：G64; O6

\section{A Comprehensive Chemical Experiment on Synthesis and Thermal Stability Study of Hybrid Perovskite Photovoltaic Materials}

Ping Che *, Han Yang, Huili Fan, Mingwen Wang, Wenjun Li

Department of Chemistry, University of Science and Technology Beijing, Beijing 100086, P. R. China.

\begin{abstract}
In this paper, a comprehensive chemical experiment is introduced, including the preparation of organicinorganic hybrid perovskite photovoltaic materials by one-step solution method. The characterization of crystal structure and morphology, the test of UV-Vis absorption spectrum, and the study of thermal stability of the obtained materials were carried out.
\end{abstract}

Key Words: Hybrid perovskite; Photovoltaic materials; Synthesis; Thermal stability;

Comprehensive experiment

近年来, 随着环境污染的加剧和化石能源资源日益减少, 太阳能将与其他新型能源(氢能、生物 质能、风能、地热能、潮汐能等)一起成为石油、煤、天然气等不可再生能源的理想补充和替代。如 今太阳能电池的应用已从军事、航天领域逐渐进入到工业、农业、商业以及公用设施等领域。但是, 要实现光伏发电社会化应用, 使太阳能电池等成为能源的主要组成部分, 目前仍存在一些问题, 如 原材料成本高、制备过程能耗大、污染严重等。降低电池成本, 除了通过现有电池产品生产的标准 化、自动化和规模化来考虑外, 从研发的角度主要通过两种途径解决, 一是降低现有电池生产成本, 主要是降低原材料与能耗的成本; 二是提高太阳能电池的光电转换效率, 使电池有高的性能价格比。 2009 年, 日本桐荫横滨大学宫坂力(Miyasaka)教授课题组首次将有机-无机杂化的钻钛矿材料应用于 太阳能电池的研究 ${ }^{11]}$, 经过近十年的发展, 钙钛矿太阳能电池的光电转化效率已经从 $3.8 \%$ 提高到 $23.2 \%$ [2]。研究者发现这种化学式为 $\mathrm{CH}_{3} \mathrm{NH}_{3} \mathrm{PbX}$ ( $\left.\mathrm{X}=\mathrm{Cl}, \mathrm{Br}, \mathrm{I}\right)$ 的钙铁矿结构化合物几乎拥有完美 太阳能电池材料的所有条件: 合适的直接带隙、高的吸收系数、优异的载流子输运性能、高的缺陷 容忍度、温和的合成条件以及不含稀有元素及贵金属等。但这种钙钛矿材料也有一些缺点, 如含有

收稿：2019-04-03; 录用：2019-06-13; 网络发表：2019-06-28

”通讯作者, Email: cheping@ustb.edu.cn

基金资助: 教育部 2018 年新工科研究与实践研究项目; 分析传感技术与信息方法学专业建设研究与实践(新工科专业改革类项目); 北京科技大

学教育教学改革重点项目(JG2017Z01) 
$\mathrm{Pb}$ 且容易热分解等 ${ }^{[3]}$ 。但随着研究的不断推进, 相信不久的将来, 这些材料的缺陷会被改进, 钙钛 矿太阳能电池可望获得实用化。为了增进化学系本科生对这一前沿研究的了解, 我们将杂化钙钛矿 太阳能电池材料的合成及热稳定性研究的综合化学实验引入大学课堂, 合成 $\mathrm{CH}_{3} \mathrm{NH}_{3} \mathrm{PbX}_{3}(\mathrm{X}=\mathrm{Cl}$,

$\mathrm{Br} ， \mathrm{I})$ 及其衍生物，并利用差示扫描量热计(DSC)研究这些杂化材料的热稳定性。

\section{1 实验目的}

(1) 初步了解太阳能电池材料的前沿进展。

(2) 掌握钙钛矿型有机-无机杂化材料的化学合成方法。

(3) 掌握利用 DSC 进行反应热测量的方法。

(4) 初步掌握有机-无机杂化材料的热稳定性研究方法。

\section{2 实验原理}

\section{1 钻钦矿型有机-无机杂化材料的合成}

本实验采用 $\mathrm{CH}_{3} \mathrm{NH}_{3} \mathrm{X}$ 和 $\mathrm{PbX}_{2}$ (X 为卤素)在 $\mathrm{DMF}$ 中反应合成 $\mathrm{CH}_{3} \mathrm{NH}_{3} \mathrm{PbX}_{3}$, 反应过程如下:

$\mathrm{CH}_{3} \mathrm{NH}_{3} \mathrm{X}+\mathrm{PbX}_{2} \stackrel{\text { DMF. }}{\longrightarrow} \mathrm{CH}_{3} \mathrm{NH}_{3} \mathrm{PbX}_{3}$

产物为钙钛矿结构, 其中有机部分为 $\mathrm{CH}_{3} \mathrm{NH}_{3}^{+}$离子, 无机部分为 $\mathrm{Pb}-\mathrm{X}$ 八面体。 $\mathrm{Pb}$ 位于八面体 的中心, $\mathrm{X}$ 位于八面体的六个顶角, 相邻八面体以共顶点相连, 有机阳离子团位于八面体空隙中, 如图 1 所示。 $\mathrm{A}$ 为有机阳离子, $\mathrm{B}$ 为过渡金属阳离子, $\mathrm{X}$ 为氧离子或卤素。

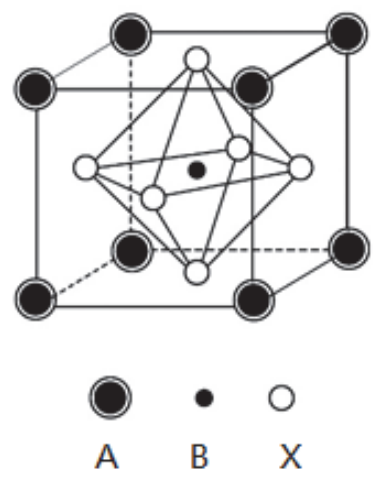

图 1 钻钛矿的晶体结构

由于三个位置均可用其他离子取代, 且取代后可引起晶体结构和分子极性的变化, 从而影响材 料的性质。根据化学基本原理, 可设计制备不同的材料, 并有希望获得具有更高热稳定性的新材料, 有兴趣的学生可以尝试。

\section{2 热效应的测量}

化学反应多伴随有热量的吸收或释放, 准确测量化学反应热是研究化学反应原理及材料热稳定 性的重要方法。在无机化学中, 我们学习了利用弹式量热计测量化学反应等容热效应的原理和方法。 目前, 在实验室中, 人们最常用的测量反应热的方法是差热分析法(DTA)和差式扫描量热法 (DSC)。 其中 DSC 方法是现在准确测量化学反应热效应的主要手段。将有物相变化的样品和在所测定温度范 围内不发生相变且没有任何热效应产生的参比物, 在相同的条件下进行等温加热或冷却, 当样品发 生相变时, 在样品和参比物之间就产生一个温度差。放置于它们下面的一组差示热电偶即产生温差 电势 $U \Delta T$, 经差热放大器放大后送入功率补偿放大器, 功率补偿放大器自动调节补偿加热丝的电流, 使样品和参比物之间温差趋于零, 两者温度始终维持相同。此补偿热量即为样品的热效应, 以电功 率形式显示于记录仪上。

功率补偿型的 DSC 是内加热式, 用来装样品和参比物的支持器是各自独立的元件, 在样品和参 
比物的底部各有一个加热用的铂热电阻和一个测温用的铂传感器。它是采用动态零位平衡原理, 即 要求样品与参比物温度, 无论样品吸热还是放热时都要维持动态零位平衡状态, 也就是要保持样品 和参比物温度差趋向于零。DSC 测定的是维持样品和参比物处于相同温度所需要的能量差 $(\Delta W=$ $\mathrm{d} H / \mathrm{d} t)$, 反映了样品的焓变。

热流型 DSC 是外加热式, 通过空气和康铜做的热垫片两个途径把热传递给试样杯和参比杯, 试 样杯的温度由镍铬丝和镍铝丝组成的高灵敏度热电偶检测得到, 参比杯的温度则由镍铬丝和康铜组 成的热电偶加以检测。由此可知, 检测的是温差 $\Delta T$, 它是试样热量变化的反映。

\section{3 实验药品及仪器}

实验原料与试剂见表 1 。

表 1 实验原料与试剂

\begin{tabular}{ccc}
\hline 药品 & 纯度 & 生产厂家 \\
\hline $\mathrm{CH}_{3} \mathrm{NH}_{3} \mathrm{Cl}$ & $97 \%$ & 国药集团化学试剂有限公司 \\
$\mathrm{CH}_{3} \mathrm{NH}_{3} \mathrm{I}$ & $97 \%$ & 国药集团化学试剂有限公司 \\
$\mathrm{DMF}$ & 分析纯 & 国药集团化学试剂有限公司 \\
$\mathrm{PbCl}_{2}$ & 分析纯 & 天津光复化工精细研究所 \\
$\mathrm{PbI}_{2}$ & 分析纯 & 天津光复化工精细研究所 \\
氯化亚锡 & 分析纯 & 天津光复化工精细研究所 \\
异丙醇 & 分析纯 & 国药集团化学试剂有限公司 \\
\hline
\end{tabular}

实验仪器: 天平, 三角烧瓶, 磁力摚拌器, 回流装置(实验室搭建), 旋蒸仪, 紫外-可见光谱仪 (UV2600, 天美, 中国), XRD 粉末衍射的仪器 (X'pert RPO, PANalystical Cooperation, 荷兰), 差 示扫描量热仪(DSC Q2000，TA，美国)。

\section{4 实验步骤}

\section{1 样品制备}

将 $\mathrm{MX}_{2}(\mathrm{M}=\mathrm{Pb}, \mathrm{Sn})$ 和 $\mathrm{CH}_{3} \mathrm{NH}_{3} \mathrm{X}(\mathrm{X}=\mathrm{Cl}, \mathrm{I})$ 按一定化学计量比(通常为 $1: 3$, 保证 $\mathrm{CH}_{3} \mathrm{NH}_{3} \mathrm{X}$ 过 量) 溶解在溶剂中得到前驱体溶液, 按不同的物质的量比加入金属卤化物, 制备混合钙钛矿材料。在 锥形瓶中电磁搅拌 $30 \mathrm{~min}$, 连接回流装置并在 $120{ }^{\circ} \mathrm{C}$ 回流 $30 \mathrm{~min}$, 此时 $\mathrm{CH}_{3} \mathrm{NH}_{3} \mathrm{X}$ 将与 $\mathrm{PbX}_{2}$ 依反 应方程式(1)反应并得到目标产物 $\mathrm{CH}_{3} \mathrm{NH}_{3} \mathrm{PbX}_{3}$ 。过量的 $\mathrm{CH}_{3} \mathrm{NH}_{3} \mathrm{X}$ 仍然溶解在溶剂中。将烧瓶转移 至旋蒸仪上, $160{ }^{\circ} \mathrm{C}$ 蒸至近干, 此时过量的 $\mathrm{CH}_{3} \mathrm{NH}_{3} \mathrm{X}$ 随溶剂蒸出。转移至真空干燥箱中, $160{ }^{\circ} \mathrm{C}$ 烘干 $1 \mathrm{~h}$ 得到目标化合物固体, 称量所制备的材料并计算产率 ${ }^{[4-6]}$ 。

\section{2 样品稳定性测试}

试样准备: 取样并称好重量, 选择样品盘类型以及相应模具, 压好。金属类样品取样量小于 $5 \mathrm{mg}$ 。高分子聚合物取样量一般选择 $10 \mathrm{mg}$ 。混合物取样量一般大于 $10 \mathrm{mg}$ 。样品尽量薄并覆盖样品 底部。

测试条件: 载气为高纯氮气, 出口压力为 $0.1 \mathrm{MPa}$ 。温度范围为室温至 $300{ }^{\circ} \mathrm{C}$, 升温速率为 $10{ }^{\circ} \mathrm{C} \cdot \min ^{-1}$ 。

把压好的样品和同类型的参比盘放入炉体中。按本实验所采用的热电(TA)公司的 DSC Q2000 的 测试步骤进行测试条件、样品信息及存储路径等参数进行设定, 并在实验完成后, 取出样品, 保存 数据, 按关机程序关闭仪器及计算机。 


\section{5 结果与讨论}

图 2 所示为所制备材料的晶体形貌。从图 2a 中可以看出, 所制备的材料大多呈多面体结构, 表 面光滑。颜色为较深的黑色, 说明该材料对可见光具有很强的吸收。在扫描电子显微镜下观察晶体 的表面(图 2b)，可发现所制备材料的表面呈岛状结构。

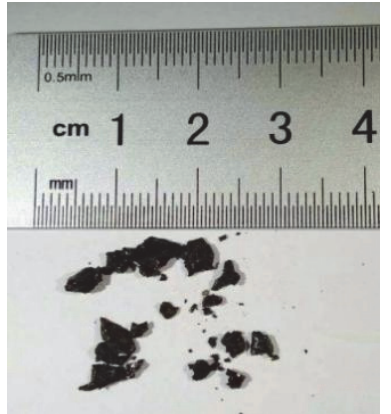

(a)

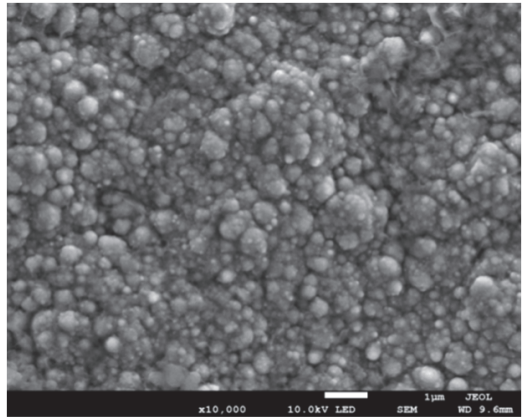

(b)

图 2 样品 $\mathrm{CH}_{3} \mathrm{NH}_{3} \mathrm{PbI}_{3}$ 的形貌

(a) 样品外观; (b) 微观结构

从图 3 可以看出, 所制备的钙钛矿材料在可见光区存在较强吸收, 吸收范围覆盖了几乎整个可 见光区域(450-800 nm)，其最强吸收区域出现在 500-700 nm，非常适合作为光吸收材料。

图 4 是所制备材料的 X 射线衍射图, 从图中可以看出, 所制备材料的衍射峰尖锐, 说明材料结 晶完好。主要衍射峰均可归属于四方钙钛矿结构, 空间群为 $P m-3 m^{[7]}$ 。说明所制备的材料符合我们 的预期, 是一种典型的有机-无机杂化的钙钛矿结构。没有发现原料的衍射峰, 也没有发现任何与 $\mathrm{Pb}$ 有关的其他化合物的衍射峰, 说明所制备的材料为纯的 $\mathrm{CH}_{3} \mathrm{NH}_{3} \mathrm{PbI}_{3}$ 钙钛矿材料。

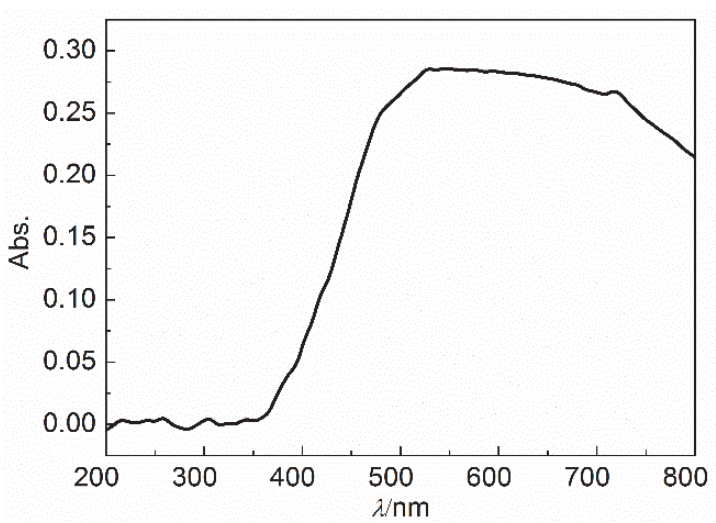

图 3 样品 $\mathrm{CH}_{3} \mathrm{NH}_{3} \mathrm{PbI}_{3}$ 的紫外-可见吸收光谱

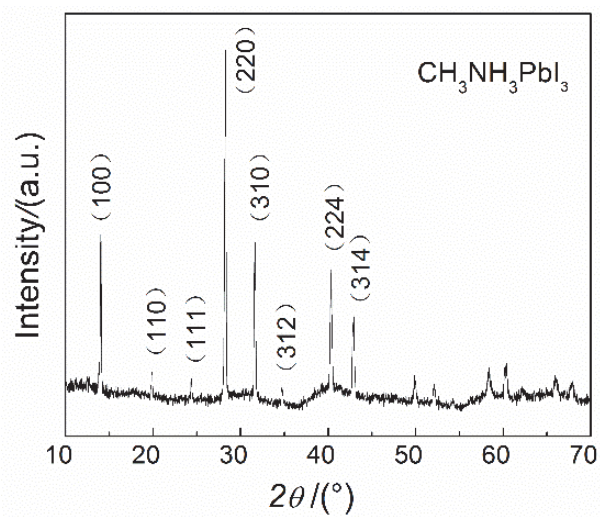

图 4 样品 $\mathrm{CH}_{3} \mathrm{NH}_{3} \mathrm{PbI}_{3}$ 的 XRD 衍射图

图 5 为所制备的样品的 DSC 曲线。根据实验条件的不同, 所制备的有机-无机杂化钙钛矿材料 在室温至 $300{ }^{\circ} \mathrm{C}$ 范围内存在一次相变和一次热分解 ${ }^{[7]}$ 。位于 $140{ }^{\circ} \mathrm{C}$ 的放热峰归属于四方相向立方 相转变。位于 $275^{\circ} \mathrm{C}$ 的放热峰可归属于钲钛矿材料的分解。

\section{6 思考}

根据 DSC 结果计算所制备的钙钛矿材料分解反应的焓变, 推测可能的反应产物, 查表计算理论 焓变并与实验结果进行比较分析。通过查阅资料分析材料由四方晶系转变为立方晶系的相变温度及 能量变化。

通过与标准卡片比对, 初步判断所制备的材料的纯度。如果出现杂质相, 通过卡片数据判断杂 质的种类。思考产生杂质的可能原因。 


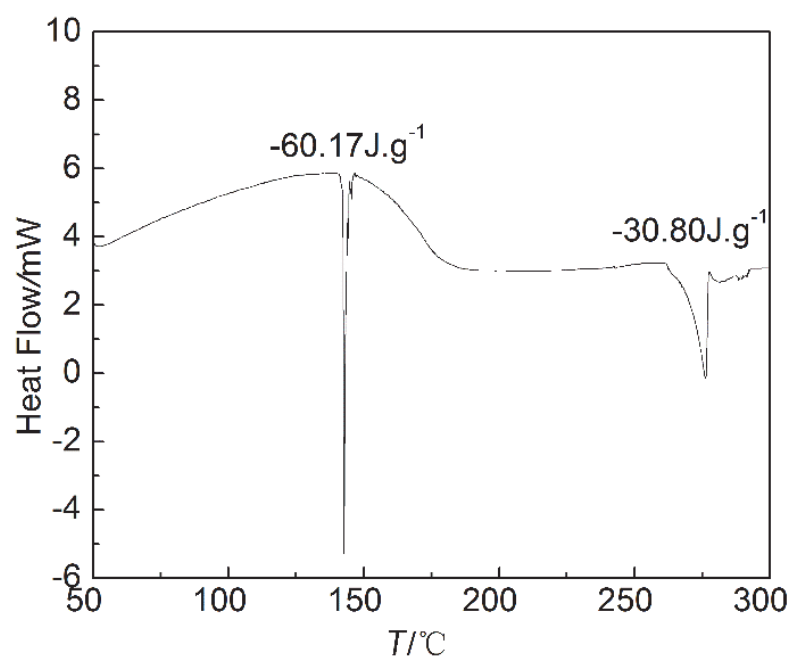

图 5 样品 $\mathrm{CH}_{3} \mathrm{NH}_{3} \mathrm{PbI}_{3}$ 的 DSC 分析结果

\section{7 教学特点}

该实验是我系为应用化学专业大二学生开设的 “科研方法入门” 课程的实验课, 经过三年的教 学实践, 发现该实验对学生了解和掌握化学实验方法、了解化学材料的科技前沿发展取得了较好的 效果。尤其是对无机化学和物理化学课程中讲述的化学反应热效应测量的理论知识的巩固、对以功 能为导向的材料设计及化学合成的新理念的建立以及对新型绿色能源材料化学合成兴趣的培养等, 都起到了十分重要的作用。实验采用每人一组的形式。学时分配为合成 2 学时、表征 8 学时。由于 本材料的无机离子既可以选择 $\mathrm{Pb}$, 又可以选择 $\mathrm{Sn}$, 卤素可选择 $\mathrm{Cl} 、 \mathrm{Br} 、 \mathrm{I}$ 三种, 因此可将学生按不 同组合分为六个大组，每组做一种材料，实验结束后可进行交叉讨论。

由于本实验是为二年级学生设计的, 加上学时的限制, 内容还不够全面。对于高年级的学生, 本实验可加入自主设计的部分, 在材料表征方面, 也可加入红外光谱分析及光电转化效率的测量及 分析等。

\section{8 结语}

有机-无机钙钛矿材料作为一种新型太阳能电池材料, 近年来获得了人们广泛的关注。随着研究 的深入, 其光电转化效率已达到 $23.2 \%$ 。但该材料存在的热稳定性差的缺点一直是制约材料走向实 用化的瓶颈。本实验利用不同比例的卤素来改变钙钛矿中 $\mathrm{Pb}-\mathrm{X}$ 八面体的结构, 从而研究其对材料 的热稳定性的影响, 具有重要的理论意义。将本实验引入大学实验课堂, 可激励学生瞄准前沿课题, 利用所学的化学知识, 解决人们所关心的材料问题。

\section{参 考 文 献}

[1] Kojima, A.; Teshima, K.; Shirai, Y.; Miyasaka, T. J. Am. Chem. Soc. 2009, 131 (17), 6050.

[2] Jeon, N. J.; Na, H.; Jung, E.-H.; Yang, T.-Y.; Lee, Y. G.; Kim, G.; Shin, H.-W.; Seok, S.; Lee, J.; Seo, J. Nat. Energy 2018,3 , 682.

[3] Hodes, G. Science 2013, 342, 317.

[4] Im, J.-H.; Lee, C.-R.; Lee, J.-W.; Park, S.-W.; Park, N.-G. Nanoscale 2011, 3 (10), 4088.

[5] Kim, H.-S.; Lee, C.-R.; Im, J.-H.; Lee, K.-B.; Moehl, T.; Marchioro, A.; Moon, S.-J.; Humphry-Baker, R.; Yum, J.-H.; Moster, J. E.; Grätzel, M.; Park, N.-G. Sci. Rep. 2012, 2, 591.

[6] Zhao, Y. X.; Zhu, K. J. Phys. Chem. C 2014, 118 (18), 9412.

[7] Chen, X.; Li, N.; Li, Y.; Che, P. Mater. Lett. 2019, 235, 239. 